Revista de

Estudios

Kantianos

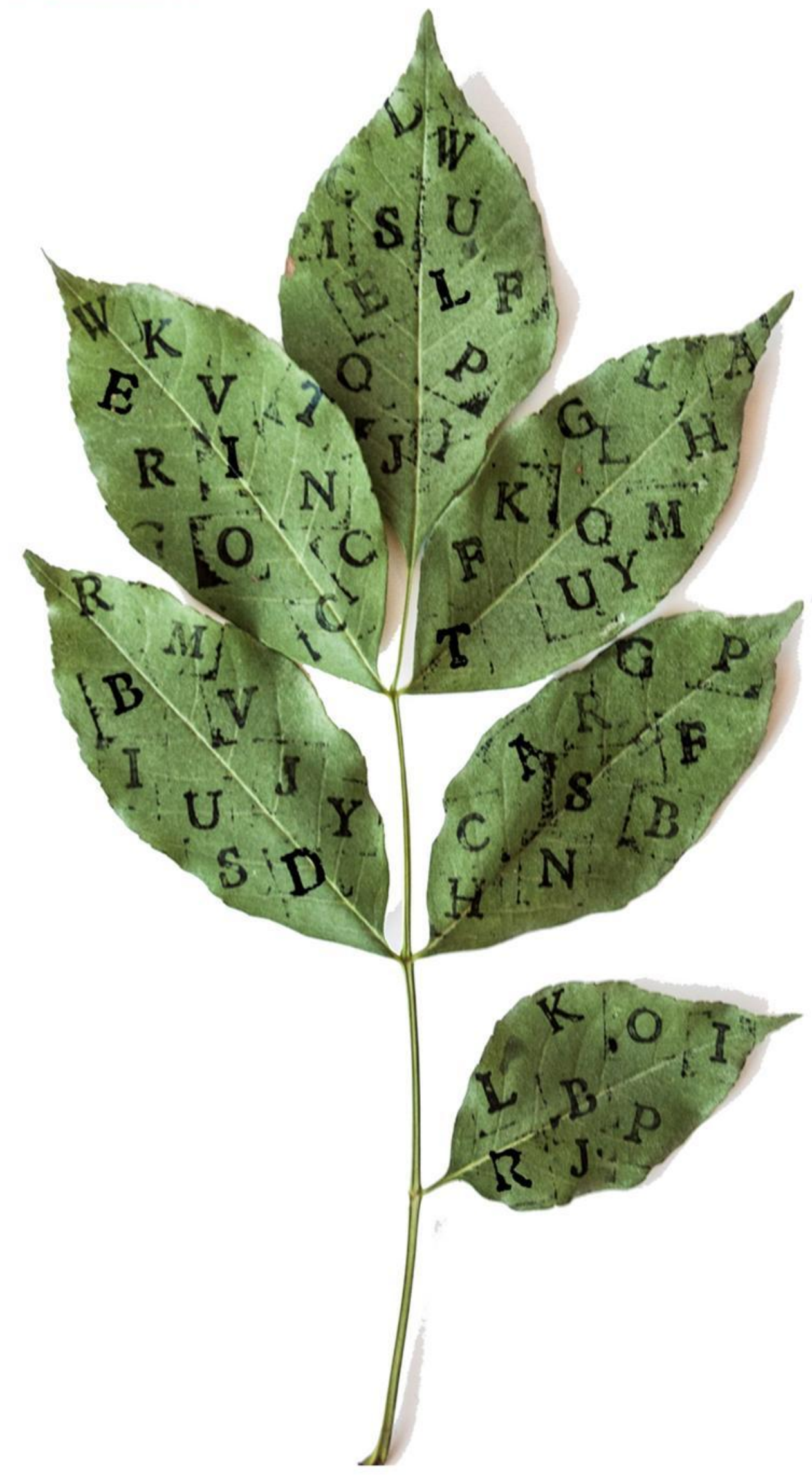


Revista de Estudios Kantianos 


\section{Revista de Estudios Kantianos}

Publicación internacional de la Sociedad de Estudios Kantianos en Lengua Española Internationale Zeitschrift der Gesellschaft für Kant-Studien in Spanischer Sprache International Journal of the Society of Kantian Studies in the Spanish Language

\section{Dirección}

Pedro Jesús Teruel, Universitat de València

pedro.teruel@uv.es

Hernán Pringe, CONICET-Universidad de Buenos Aires/

Universidad Diego Portales, Santiago de Chile

hpringe@gmail.com

\section{Secretario de edición}

Fernando Moledo, CONICET-Universidad de Buenos Aires

fernandomoledo@filo.uba.ar

\section{Secretaria de calidad}

Marcela García, Universidad de Morelia, México

garciar.marcela@gmail.com

\section{Editores científicos}

Jacinto Rivera de Rosales, UNED, Madrid

Claudia Jáuregui, Universidad de Buenos Aires

Vicente Durán, Pontificia Universidad Javeriana, Bogotá

Julio del Valle, Pontificia Universidad Católica del Perú, Lima

Jesús Conill, Universitat de València

Gustavo Leyva, Universidad Autónoma de México, México D. F.

María Xesús Vázquez Lobeiras, Universidade de Santiago de Compostela

Wilson Herrera, Universidad del Rosario, Bogotá

Pablo Oyarzun, Universidad de Chile, Santiago de Chile

Paula Órdenes Azúa, Universität Heidelberg 


\section{Comité científico}

Juan Arana, Universidad de Sevilla

Reinhardt Brandt, Philipps-Universität Marburg

Mario Caimi, Universidad de Buenos Aires

Monique Castillo, Université de Paris-Est

Adela Cortina, Universitat de València

Bernd Dörflinger, Universität Trier

Norbert Fischer, Universität Eichstätt-Ingolstadt

Miguel Giusti, Pontificia Universidad Católica del Perú

Dulce María Granja, Universidad Nacional Autónoma de México

Christian Hamm, Universidad Federal de Santa María, Brasil

Dietmar Heidemann, Université du Luxembourg

Otfried Höffe, Universität Tübingen

Claudio La Rocca, Università degli Studi di Genova

Juan Manuel Navarro Cordón, Universidad Complutense, Madrid

Carlos Pereda, Universidad Nacional Autónoma de México

Gustavo Pereira, Universidad de la República, Uruguay

Ubirajara Rancan de Azevedo, Universidade Estadual Paulista, Brasil

Margit Ruffing, Johannes Gutenberg-Universität Mainz

Gustavo Sarmiento, Universidad Simón Bolívar, Venezuela

Sergio Sevilla, Universitat de València

Roberto Torretti, Universidad Diego Portales, Santiago de Chile

Violetta Waibel, Universität Wien

Howard Williams, University of Aberystwyth

Allen W. Wood, Indiana University

Diseño, editor de estilo y maqueta

Josefa Ros Velasco, Universidad Complutense de Madrid

\section{Entidades colaboradoras}

Sociedad de Estudios Kantianos en Lengua Española (SEKLE)

Departament de Filosofia de la Universitat de València 


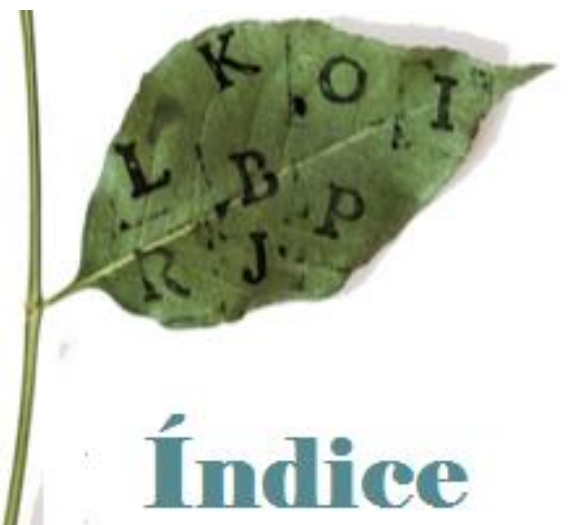

\section{Artículos}

9 Pensar como operación - Acerca de los presupuestos e implicaciones de la lógica formal moderna

Max Gottschlich

DOI 10.7203/REK.2.1.10059

20 Los conceptos kantianos de "facultad" y de "mente" frente a la lectura epigenética Antonino Falduto

DOI 10.7203/REK.2.1.10011

29 Arqueología filosófica y hermenéutica. Apuntes sobre la interpretación de la historia de la filosofía en Kant

José García Gómez del Valle

DOI 10.7203/REK.2.1.8811

48 Continuidad y novedad en la concepción histórica de Kant en El conflicto de las facultades

Natalia Lerussi

DOI 10.7203/REK.2.1.9892

60 It's impossible to will to be punished? Exploring consensual way out of the Kantian dilema Matías Parmigiani

DOI 10.7203/REK.2.1.8813

\section{Recensiones}

89 Immanuel Kant (trad. Alba Jiménez): Lecciones de filosofía moral Mrongovius II.

Salamanca, Sígueme, 2017, 160 pp. ISBN: 978-8-4301-1954-7

Pablo Veraza Tonda

DOI 10.7203/REK.2.1.9990 
91 Faviola Rivera Castro: Virtud, Felicidad y Religión en la Filosofía Moral de Kant. México, Instituto de Investigaciones Filosóficas-UNAM, 2014, 328 pp. ISBN: 978-607-02-4788-0

Gustavo Leyva

DOI 10.7203/REK.2.1.9992

93 Vicente de Haro Romo: Duty, Virtue and Practical Reason in Kant's Metaphysics of Morals. Hildesheim/Zürich/New York, Georg Olms Verlag, 2015, 342 pp. ISBN: 978-3487-15348-3

Eduardo E. Charpenel

DOI 10.7203/REK.2.1.9998

96 Paolo Grillenzoni: Kant e la scienza (1755-1760). Roma, Aracne, 2016, 575 pp. ISBN: 9788-854-89523-2

Daniele Savino

DOI 10.7203/REK.2.1.10054

100 Nuria Sánchez Madrid: A Civilização como destino. Kant e as formas da reflexão.

Florianópolis, Nefiponline, 2016, 288 pp. ISBN: 978-85-99608-16-6

Josefa Ros Velasco

DOI 10.7203/REK.2.1.9801

103 Laura Anna Macor: Die Bestimmung des Menschen (1748-1800): Eine Begriffsgeschichte. Forschungen und Materialien zur deutschen Aufklärung. Abteilung II: MonographienFMDA II, 25. Stuttgart-Bad Cannstatt, Frommann-Holzboog, 2013, 432 pp. ISBN: 978-37728-2615-3

Daniel Schwab

DOI 10.7203/REK.2.1.9989

\section{Informes}

109 Rezension zur Internationalen Tagung "Teleologische Reflexion in Kants Philosophie" Anna Pickhan (geb. Brechtel)

DOI 10.7203/REK.2.1.9993

\section{Entrevistas}

112 Entrevista a Pablo Muchnik

Fernando Moledo

DOI 10.7203/REK.2.1.10064

\section{Convocatorias y normas para autores}

118 Kant en México

DOI 10.7203/REK.2.1.10085

119 II Premio Kant para Jóvenes Investigadores e Investigadoras DOI 10.7203/REK.2.1.10086 
120 IV Congreso Internacional de la SEKLE, Valencia, 2018 DOI 10.7203/REK.2.1.10088

121 Normas para autores DOI 10.7203/REK.2.1.10091 


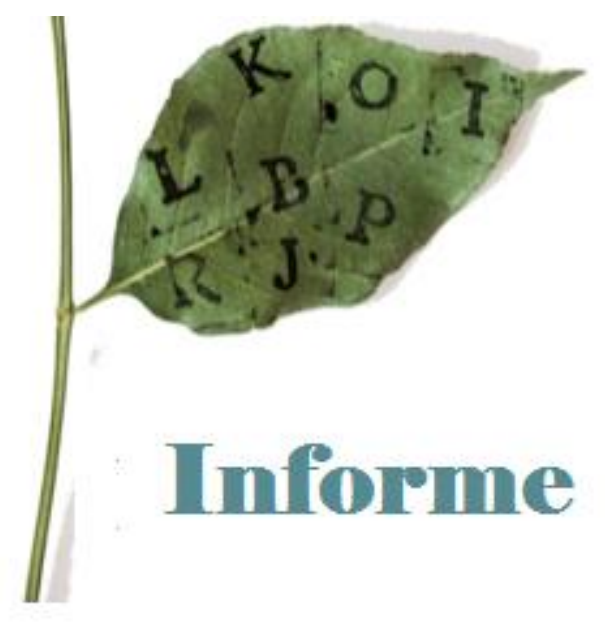




\title{
Rezension zur Internationalen Tagung „Teleologische Reflexion in Kants Philosophie"
}

\author{
ANNA PiCKHAN (GEB. BRECHTEL) $)^{1}$
}

Am 10. und 11. Februar 2017 fand am Philosophischen Institut der Universität Heidelberg die von Paula Órdenes und Daniel Schwab organisierte Internationale Tagung „Teleologische Reflexion in Kants Philosophie" statt. Ziel war eine Auseinandersetzung mit den unterschiedlichsten Ansätzen, die Immanuel Kants Konzeptionen von Teleologie betreffen. So waren die Beiträge inhaltlich sehr weit gefächert.

Peter McLaughlin und Peter König vom Philosophischen Seminar in Heidelberg eröffneten mit ihren Vorträgen am Freitagnachmittag die Veranstaltung.

Das Anliegen McLaughlins war es in seinem Vortrag „Freiheit und technisch-praktische Vernunft bei Kant" Probleme bei der kantischen Freiheitskonzeption aufzuwerfen. In seiner sehr umfassenden Betrachtung (Ausführungen zur Handlungstheorie und Philosophie der Biologie, der poiesis und praxis, dem Begriff der Verantwortung, der Empirischen Freiheit u.v.m.) machte er u.a. darauf aufmerksam, dass Kant mit seinem Ansatz von ausschließlich kausalen Handlungserklärungen gegenüber Locke und Leibniz sogar rückständig sei, weil ihm die Sphäre rationaler Handlungsbegründungen fehle. Daraus erwüchsen viele Probleme im Bereich der Freiheitskonzeption, die McLaughlin als abschließende Fragen an Kant formulierte.

Das Anliegen von Peter König mit seinem Vortrag „Teleologische Kriterien materialer Wahrheit. Zur Theorie des Gemeinsinns bei Kant" war systematischer Art. Die Stelle der Kritik der Urteilskraft im System der kantischen Kritiken sowie der systematische Zusammenhang von ästhetischer und teleologischer Urteilskraft sollten aufgezeigt werden. Mit Hilfe einer neuen Deutung des ,unum verum bonum” aus $\$ 12$ der Kritik der reinen Vernunft konnte er deutlich machen, dass es in der Kritik der Urteilskraft, entgegen der einfachen Schulmeinung, auch um Wahrheitserkenntnis, i.S. der Bedingungen wahrer Erkenntnis des empirisch Gegebenen, gehe. Für die Systematik innerhalb der Kritik der Urteilskraft sei der Gemeinsinn entscheidend, weil er im Gegensatz zum System der Natur (der objektiven Seite der Bedingungen der Möglichkeit empirischer Urteile) die subjektive Seite liefere.

Anna Brechtel (Jena) vertrat mit ihrem Beitrag zur Körperkonzeption „Der Körper in Kants Opus postumum. Einige teleologische Implikationen" die These, dass durch den Körper, so wie ihn Kant in seinem Nachwerk fasst, eine neue Dimension des Übergangs zwischen Metaphysik und Physik in teleologischer Absicht geschaffen werde. Der Körper forme dort ein teleologisches Prinzip, welches der Urteilskraft einen Leitfaden zur Erschließung der Natur an die Hand gebe. Neben dieser konkreten Anweisung für die Methode des Naturforschers wird qua unserer eigenen Körperlichkeit auch der Wahrheitswert der Naturerkenntnis auf eine neue Weise gefasst: Wir Menschen bekommen nur das aus der Natur heraus, was wir zuvor in sie hineingelegt haben.

Mit dem Vortrag „Die Antinomie der teleologischen Urteilskraft und Kants Auflösung im Licht der Einleitung in der Kritik der Urteilskraft" unternahm Daniel Schwab (Heidelberg) einen eigenen Versuch der Interpretation der Antinomienproblematik in der Kritik der Urteilskraft. Mit einem Durchgang durch die einschlägigen Paragraphen ( $§ 70-78$ der Dialektik) erklärte er, dass kein Widerspruch zwischen den Maximen zu finden sei. Der bei vielen Interpreten noch immer umstrittene Schein bestehe allerdings gerade im antinomischen Anschein eines Widerspruchs zwischen den

\footnotetext{
${ }^{1}$ Friedrich-Schiller-Universität Jena. Anna.brechtel@ uni-jena.de
} 
Maximen der reflektierenden Urteilskraft. Dem könne man sich jedoch nicht entziehen, da er qua unserer eigenen teleologischen Beurteilungsweise immer bestünde.

Am Samstag Vormittag kam Georg Toepfer (Berlin) in seinem Vortrag „Kants Teleologie heute" auf vier verschiedene Themenbereiche zu sprechen, die bei Kant mit Teleologie in Verbindung gebracht werden: Biologie, Ökologie, Anthropologie und Geschichtsphilosophie. Interessante Perspektiven eröffneten sich, als Toepfer zeigte, welche Aktualität die kantischen Ansätze in diesen Bereichen heutiger wissenschaflicher Debatten haben. Zum Schluss seiner Erarbeitungen konstatierte er Kant eine Einheit in der Teleologie-Konzeption selbst. So seien vor allem zwei Bereiche auszumachen, die, obwohl sehr unterschiedlich, doch voneinander abhängen: der natural-zyklische (mit der Biologie und Ökologie) sowie der kulturell-lineare (mit der Anthropologie und der Geschichtsphilosophie).

Manuel Sánchez Rodríguez (Granada) trug über das Thema „Kants Theorie der reflektierenden Urteilskraft als eigentliche Apologie (und Kritik) der leibnizschen Prästabilierten Harmonie" vor. Sein Anliegen war es mit dem Leibnizianer Johann Augustus Eberhard zu zeigen, dass die Kantrezeption nie nur werkimmanent stattfinden sollte, sondern vielmehr Kants Auseinandersetzung mit seinen Vorläufern sowie Zeitgenossen für sein Schaffen berücksichtigt werden muss. Historisch sehr genau machte er deutlich, dass die Theorie der reflektierenden Urteilskraft am besten durch Kants kritische Revision von Leibniz prästabilierter Harmonie zwischen Substanzen zu erklären sei, die dieser durch die Idee eines freien Spiels zwischen den Erkenntniskräften ersetzt.

Ina Goy (Tübingen) unternahm in ihrem Vortrag „Why animals let man belive in God” den Versuch Teleologie mit Kants generellen Äußerungen über Tiere in Verbindung zu bringen. Sie zeigte, dass Tiere Menschen dazu bringen an Gott zu glauben, damit die Einheit zwischen der natürlichen und moralischen Ordnung innerhalb und zwischen Tieren gewährleistet werden kann. Die Idee von Gott sei dabei einheitsstiftend für mechanische und physiko-teleologische Kräfte und Gesetze genauso wie für physiko-teleologische und moralische Kräfte und Gesetze. Insofern sei der Mensch selbstverständlich als Teil des tierischen Kosmos zu sehen.

Eine weitere Hauptfrage der Teleologie ist zweifelsohne die Frage nach dem Zweck der Schöpfung. Fernando Moledo (Buenos Aires) ging in seinem Beitrag „Der kantische Begriff des Menschen als Zweck der Schöpfung: verschiedene Argumente um eine grundlegende Idee” vor allem auf die Frage ein, warum gerade das Moralisch-Sein den Menschen zum Zweck der Schöpfung macht. Mit Hilfe von Kants Anthropologie sowie dessen Moralphilosophie führte Moledo aus, dass es die vernünftige Natur des Menschen sei, die denselben zum Zweck der Schöpfung macht. Nur dadurch könne ein freier Wille garantiert werden und durch die Gleichsetzung von frei sein und unter sittlichen Gesetzen stehen sei schließlich der moralische Mensch als Zweck der Schöpfung bei Kant gezeigt.

Dann beschäftigte sich Paula Órdenes (Heidelberg) in ihrem Vortrag „Teleologische Einheit der Vernunft?" mit der einheitsstiftenden Funktion der Vernunft. Mittels eines Durchgangs durch die Kritik der reinen Vernunft, die Kritik der praktischen Vernunft und die Kritik der Urteilskraft konnte sie plausibel darstellen, dass das Bedürfnis einer Einheit aus unserer Vernunft selbst kommt. Die Einheit werde dabei weder gegeben noch deduziert sondern bestehe als notwendige Annahme für die Erforschung der Natur, die als Objekt des theoretischen sowie praktischen Bereichs zu verstehen sei.

Der letzte Vortrag am Samstag Abend stammte von Jacinto Rivera de Rosales (Madrid) mit dem Titel „Versuch einer Auslegung der teleologischen Dialektik von Kant”. Vor einer Analyse der Dialektik eröffnete er den Zuhörern eine ganz bestimmte Perspektiv auf den kantischen Text. Als Vereinigungspunkt von Natur und Freiheit seien Lebewesen, genauer ihr Leib und seine vor-reflexive Subjektivität, zu sehen. Damit ist nach einer Auslegung der Antinomie für Rivera de Rosales klar, dass so gezeigt werden kann, dass bei Lebewesen immer beide Erklärungen gefordert werden müssen: die mechanische (als objektive) und die teleologische (als Indikator dafür, was als objektiv zu erklären sei).

Insgesamt war dieses Zusammentreffen der Kantexperten ein sehr gelungener Kongress: Die Atmosphäre war jederzeit angenehm und so konnten die an die Vorträge anschließenden 
Diskussionen auch oft wertvolle, neue Perspektiven für alle Seiten eröffnen und Beiträge zur aktuellen Forschungsdebatte entstehen. 